\title{
Les propriétés de l'Actinium (Suite)
}

\author{
Par J. GODLEWSK]
}

Professeur à l'I'niversité de Lemberg ${ }^{2}$.

\section{DEUXIÈNE PARTIE}

\section{Nature du rayonnement de l'actinium.}

On sait peu de choses des rayons $\propto$ de l'actinium. Nous savons seulement qüils sont émis par l'Ac X, l'émanation et l'Ac B.

Le pouvoir de pénétration des rayons $\beta$ et $\%$ de l'actinium a été étudié par l'auteur ${ }^{27}$.

Les mesures ont été faites sur un échantillon d'Emanationskörper au moven d'un électroscope sensible. Le fond de l'électroscope (type C. T. R. Wilson) avait été enlevé et remplacé par une lame d'aluminium de 0,08 millimètres d'épaisseur, qui absorbait tous les rayons $\propto$. La coupelle plate qui contenait 2 grammes de substance active élait fermée au mojen d'une feuille mince de mica, et placéc sous l'électroscope après interposition des différents écrans absorbants.

Les résultats sont représentés par la figure 5. Les ordonnées sont les logarithmes de l'activité $\beta$ en pour 100 de l'activité $\beta$ totale, les abcisses les épaisseurs du métal absorbant.

A titre de comparaison on a également mesuré l'absorption des rayons $\beta$ de l'uranium et du radium et les résultats sont portés sur la même tigure. On roit que tous les points expérimentaux se trouvent sur des droites. Et de fait l'équation $\mathrm{I}=\mathrm{I}_{0} e^{-i \mathrm{x}}$, où $\mathrm{X}$ dé-

1. Yoir Le Radium, t. III, no 10, octobre 1906.

2. Dans la première partie de ce mémoire, le nom de l'auteur figurait, par erreur, avec le titre de professeur à l'Université de Cracovie, II. J. Godlewski vient d'être nommé récemment professeur de physique à l'Université de Lemberg. signe l'épaisseur du métal, était strictement applicable, même pour I plus petit que 1 pour 100 de $\mathrm{I}_{0}$, c'est-à-dire quand l'activité était réduite à moins du centième. Ceci indique que les rayons $\beta$ de l'actinium

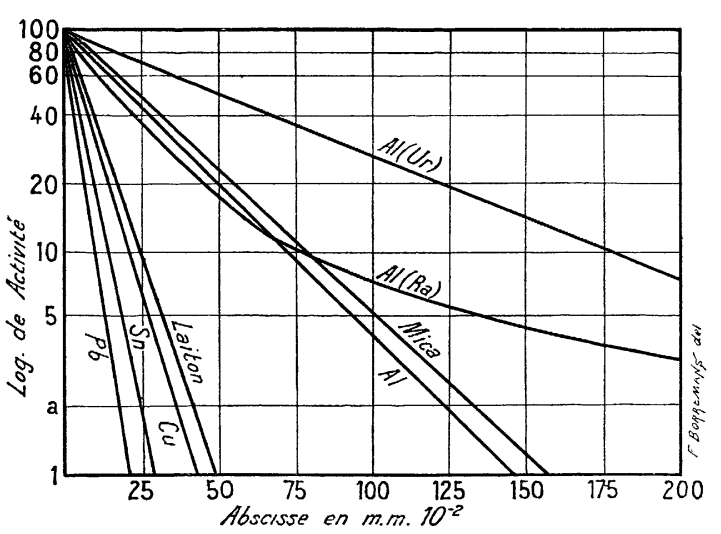

Fig. 5.

sont homogènes et que leur pouvoir de pénćtration est indépendant de la longueur qu'ils ont franchie dans le corps absorbant. Nous obtenons donc pour le coefficient d'absorption un nombre constant.

Dans le cas du radium, la figure montre qu'il en est autrement, comme l'ont montré nombre d'expérimentateurs. L'activité ne tombe pas suivant une loi exponentielle simple et le coefficient d'absorption diminue rapidement arec la distance parcourne.

Cette homogénéité des rajons $\beta$ de l'actinium a aussi été signalée récemment par I)ebierne ${ }^{29}$. Il a constaté que les rayons $\beta$ de l'actinium ne subissent aucune dispersion dans le champ magnétique, comme c'est le 
cas avec les rayons $\beta$ du radium, mais donment des bandes de deviation fines et nettes.

La scconde propriété très caractéristique des rayons $\beta$ de l'actinium est leur pouroir de pénétration relalivement faible ${ }^{27}$, car ils sont absorbés plus de deux fois plus facilement que les rayons de l'uranium et presque trois fois aussi facilement que les rayons mojens dı radium. Par contre le pouroir de pénétration des rayons du radium varie beaucoup arec la longueur franchie. La figure 5 montre qu'au début les courbes du radium et de l'actinium coïncidenl, ce qui montre que les rayons les moins pénétrants du radium sont de même nature que les rayons de l'actinium.

Les valeurs du coefficient d'absorption $\lambda$ pour les différentes substances étudićes, comme aussi les épaisseurs de ces substances qui absırbent la moitić des rayons sont rassemblées dans le tableau $\mathrm{I}$. A titre de comparaison on a mis les valeurs correspondantes pour le radium et l'uranium, telles qu'elles ont été déterminées par Rutherford et Strult.

Tableau I.

\begin{tabular}{|c|c|c|c|c|c|c|c|c|c|}
\hline \multirow{2}{*}{$\begin{array}{l}\text { SUBST 4XCE } \\
\text { absorbante }\end{array}$} & \multicolumn{3}{|c|}{ ACTINILA } & \multicolumn{3}{|c|}{ URAYIUII } & \multicolumn{3}{|c|}{ RADIUII } \\
\hline & \begin{tabular}{|c|} 
L. Ev \\
$10^{-2}$ \\
MM.
\end{tabular} & $\mid \begin{array}{l}\lambda^{-1} \\
\mathrm{cM} .\end{array}$ & $\frac{\lambda}{d}$ & \begin{tabular}{|c|} 
L Ex \\
$10^{-2}$ \\
MM.
\end{tabular} & $\begin{array}{l}\lambda^{-1} \\
\mathrm{CM}\end{array}$ & $\frac{\lambda}{d}$ & $\begin{array}{l}\text { L EX } \\
10^{-2} \\
\text { MM. }\end{array}$ & $\begin{array}{l}\lambda{ }^{-1} \\
\mathrm{CM}\end{array}$ & $\frac{\lambda}{d}$ \\
\hline Al. . & 21,2 & 32,7 & 12,9 & 49,5 & 14,0 & 5,4 & 59,7 & 11,6 & 4,30 \\
\hline Nica. & 21,0 & 33,0 & 12,0 & 40,3 & 17,2 & 5,1 & 64,2 & 10,8 & 3,94 \\
\hline Laiton . & 6,5 & 108 & 13,1 & ) & $D$ & ) & D & ) & $1)$ \\
\hline Cu. . . & 6,3 & 110 & 12,8 & 11,6 & 6,0 & 7,0 & 14,1 & 49,2 & 5,50 \\
\hline Sn . & 4,5 & 154 & 15,7 & ) & ) & )' & $D$ & ) & 1 \\
\hline Pb... & 4,25 & 163 & 14,1 & 5,6 & 12,2 & 10,8 & 11,1 & 42,5 & 5,78 \\
\hline
\end{tabular}

Les valeurs calculćes $\frac{\lambda}{d}$ ò̀ $d$ désigne la densité, montrent que la loi de Lenard sur l'absorption de masse est applicable jusqu'à un certain point, et mieux pour l'actinium que pour le radium ou l'uranium.

L'émission des rayons $\gamma$ a aussi été constatée. L'ionisation produite par les rayons $\beta$ est environ 300 fois supérieure à celle qui est due aux rayons $\gamma$.

Les mesures d'absorption des rajons $\gamma$ ont montré que l'absorption suit rigoureusement la loi exponenticlle jusqu’à ce que l'intensité soit réduite aux $50 \mathrm{p}$. 100 environ de valeur initiale. Au-dessous de cette limite les phénomènes sont trop petits pour être mesurables *. Pour voir si les rayons $\gamma$ sont pleinement ho-

\footnotetext{
* J'ai déjà signalé dans le travail cité ici cette possibilité d'une liétérogétéité des rayons $\gamma$ de l'actinium. II. Ėve, de Montréal, m'a écrit récemment qu'en se servant d'un actinium très actif, il s'est persuadé que la droite représentant les logarithmes des activités en fonction des épaisseurs traversées a un coude vers le haut au voisinage de 30 pour 100 , ee qui semble indiquer l'existence de rayons $\gamma$ plus pénétrants. Les résultats de ces recherches seront sans doute publiés bientòt.
}

mogènes ou si leur pouvoir de pénétration, comme dans le cas de radium, augmente arce l'épaisseur traversée, des mesures doivent être reprises arec un produit d'activité plus grande que celle du produit utilisé par l'auteur (environ 500 unités).

Comme les rayons $\beta$, les rayons $\%$ de l'actinium se distinguent par lcur trìs faible pouvoir de pénétration, ils sont absorbes 5 à 10 fois plus facilement que les rayons plus pénétrants du radium, comme le tableau suivant en fait foi. Pour la comparaison on a mis les nombres de Mc Clelland relatifs aux rayons $\gamma$ du radium. L désigne comme plus haut l'épaisseur du métal qui réduit le rayonnement de moitié.

\section{Tableau II.}

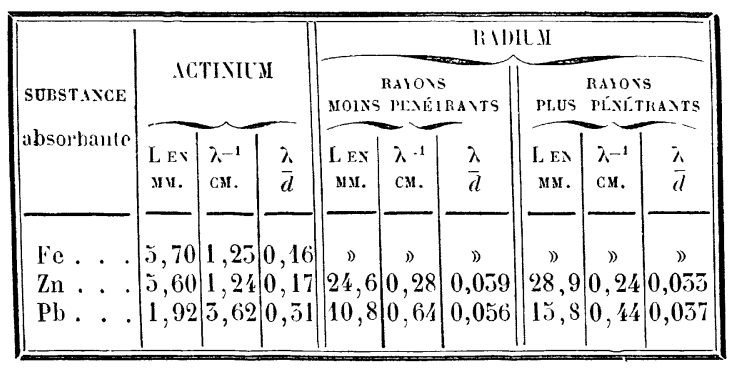

Les valeurs de $\frac{\gamma}{d}$ montrent que l'absorption des rayons $\gamma$ de l'actinium ne semble pas être proportionnelle à la densité.

\section{Production de l'hélium par l'actinium.}

Un des résultats les plus intéressants des recherches radioactives est l'obtention de l'hélium à partir de l'émanation du radium, exemple frappant de transmutation de la matière. On a d'abord admis que l'hélium était le produit de transformation ultime du radium. Mais vu la lenteur des dernières transformations du radium (celle du radium D, p. ex., comporte 40 ans), une semblable hypothèse sembla improbable. On admit done que la particule $\alpha$ elle-même pourrait ètre un atome d'hélium *. La valeur de la masse d'une"particule $\alpha$, déduit des expériences de dériation magnétique n'était pas très éloignée de la masse de l'atome d'hélium. Si vraiment la particule $\alpha$ élait l'atome d'hélium chargé positivement et projeté avec une grande vitesse, nous devrions considérer les corps radioaclifs comme des combinaisons moléculaires définies d'une substance connue ou inconnue arec l'hélium. La similitude des particules émises par différents corps semblerait indiquer qu'elles sont effeclirement de mème nalure. Il faudrait alors que tous les corps radioactifs émettent de l'hélium, en quantité proportionnelle à leur rayonnement $\alpha$.

* V. Retherforn. Radioactivuly, $2^{\circ}$ édit., 1905, p. 479. 
Or, après le radium le corps le plus actif est l'actinium. C'est donc lui qui doit de préférence nous donner de l'hélium. Et effectivement, II. Debierne a réussi l'an dernicr à montrer expérimentalement que l'actinium produit de l'hélium.

Debierne ${ }^{26}$ s'est servi pour cela de toutes ses préparations les plus actives, soit sous forme de chlorures dissous, soit sous forme de fluorures secs. La solution d'actinium, comme celle du radium, produit constamment du gaz tonnant d'activité comparable à celle du gaz qui se dégage d'une solution de radium. Le mélange des gaz de l'actinium est introduit dans un tube de verre où sont absorbés par des réactifs spéciaux les différents gaz chimiquement actifs. Debierne a cmployé pour l'artinium le même dispositif que pour le radium.

Les gaz non alssorbés sont comprimés dans un petit tube capillaire de 2 millimètres cubes environ au mojen du mercure. Ce tube porte deux électrodes de platine, et présente les raies de l'hélium avec une tclle netteté qu'il est facile de les relever photographiquement.

Les fluorures non complètement séchés qui dégagent encore du gaz tonnant, donnent aussi arec certitude de l'hélium. La quantité produite scmble comparable à cclle que fournit un radium de mème aclivité.

Dans unc expérience faite avec un sel solide d'actinium, Debierne a obtenu un spectre continu intense arec les raies de l'hydrogène et du mercure faiblement marquées. La photographie de ce spectre donne une bande parfaitement contimue qui se poursuit sans affaiblissement entre $\lambda=5 \%$ et $\lambda=650 \mu \mu$. Ce spectre curieux n’a pas encore été identifié.

On espère que Debierne publiera bientòt un compterendu détaillé de ces expériences si intéressantes et si importantes.

\section{La question Actinium-Émanium.}

Les nombreuses expériences deces dernières années ont établi que les constantes de temps des transformations radioactives caractérisent un produit donné d'une manière aussi nette que les résultats de l'analyse spectrale. Or, nous avons vu que l'actinium donne toute une série de produits de transformation très bien caractérisés, possédant chacun une constante de temps particulière. Ces constantes, les phénomènes spéciaux de phosphorescence comme aussi le rayonnement caractíristique de chaque produit successif, nous permettent de regarder l'actinium comme un ćlément noureau. Mais cette hypothèse ne se fonde que sur les études de radioactivité, les propriétés chimiques de cet élément nous demeurent entièrcment inconnues.

Nous allons nous demander maintenant si l'éma- nium est identique à l'actinium. La possibilité de cette identité a été suggérćc d'abord par Giesel luimèmes ${ }^{5}$, elle a été plus tard discutéc en détail * et ne peut plus aujourd'hui faire de doute.

Si nous désignons par actinium le corps radioactif constant qui fournit les produits de transformation énumérés plus haul possédant chacun une constante de temps caractéristique, le corps qui excite les phénomènes de phosphorescence déjà décrits et dont les rayons se distinguent par leur homogénéité et leur pouvoir de pénétration relalivement faible, il devient impossible de découvrir une seule différence entre l'émaniun et l'actinium.

La suite des produits est la même, les constantes de temps sont les mêmes. Hahn et Sackur ${ }^{22}$ ont comparé l'an dernier les constantes de temps de l'émanation et de la radioactivité induite pour l'actinium de Debierne et l'émanium de Giesel sous des conditions parfaitement identiques; les différences étaient de l'ordre des erreurs expérimentales, comme les auteurs le disent formellement. Les deux sortes de préparation excitent les mèmes phénomènes de phosphorescence, comme l'ont établi Debierne et Giesel ' lors de leur rencontre. Debicrne a récemment constaté pour les rajons $\beta$ de l'actinium la mème homogénéité que j’avais observée sur l'Emanationskörper de Giesel. Enfin M. Ève de Montréal m'a écrit qu'il a trouvé pour le coefficient d'absorption par le plomb des rayons $\gamma$ de l'actinium tout à fait le même nombre que j'arais ohtenu précédemment avec l'Emanationskörper.

Nous voyons donc qu'au point de rue radioactif, le seul qui soit décisif pour le moment, on ne peut signaler aucune différence et l'identité des deur substances s'ensuit nécessairement.

Il faut citer encore ici un travail de Marckwald ${ }^{25}$ qui s'occupe de cette question el pense la résoudre dans un sens " inattendu » et complètement différent.

Marckivald avait transiormé en chlorures les terres rares séparćes des caux-mères du radium et en arait isolé le thorium au moyen de l'hyposulfite. Ce thorium, purifié par d'autres néthodes, dégageait une orande quantité d'émanation et cette émanation disparaissait très vite. $0 \mathrm{r}$, après plusieurs mois d'observation on constate que le thorium perdait son pouroir d'émanation et d'une manière générale son activité. Les oxydes de lanthane el de didyme extraits de la solution privée de thorium ne montrèrent aucun pouvoir d'émanation sensible aprís séparation du thorium. Mais le pouvoir d'émanation reparaissait chez eux avec le temps suivant une loi complémentaire de celle suivant layuelle le thorium se désactivait.

Nous voyons donc que par précipitation à l'hyposulfite du thorium contenu dans un mélange actif de lanthane et de didyme on sépare avec le thorium un produit dont l'activité diminue avec le temps, mais qui

* Y. $14,15.16$. 
se reforme à mesure dans le mélange lanthanedidyme.

Bien que M. Marckwald, dans le mème travail, donne à ceux qui s'occupent de radioactivité le conseil de ne nommer les corps qu'après étude complète de leurs proprictés, il donne pourtant au produit séparé comme on vient de le dire un nom, le nom d'actinium, sans avoir étudié la scule propriété accessible de ce produit, son temps de transformation. Il conclut " que l'actinium et l'émanium ne sont pas iden" tiques, mais dérivent l'un de l'autre. L'émanium (" suit le lıathane et engendre l'actinium qui suit le thorium 1). Cette conclusion est inacceptable de tous points et repose sur un malentendu de nomenclature.

Comme le montrent les expériences de Harckwald, le corps qui suit le lanthane produit effectivement celui qui suit le thorium, mais ce dernier n'est pas l'actinium. Selon toute prohabilité ce corps n'est autre que l'Je $X$ qui se laisserait ainsi séparer de l'actinium en présence du thorium par précipitation à l'hyposulfite. Nous arons déjà vu que l'Ac $\mathrm{X}$ se comporte effectivement de la sorte. On pourrait aisément décider par des mesures de constante de temps si le produit de Marckwald est réellenent l'Ac X. S'il se trouvait qu'il eùt la constante de temps citée plus haut (environ 10 jours), ce serait l'Ac $X$, en dépit de la différence des traitements chimiques. Car nous appelons précisément AcX le corps qui se détruit à moitié en ce temps, qui donne avec un rayonnement caractérislique une émanation de courte durée et finalement des radioactivités induites particulières. Si au contraire le produit de Marckivald arait une constante de temps différente et des propriétés radioactives différentes de celles de l'Ac $X$, ce serait vraiment un produit nouveau qu'on pourrait désigner d'un nom quelconque, sauf celui d'actinium. Marckwald a donné à son produit le nom d'actinium parce qu'il suit le thorium dans toutes ses réactions et qu'on a trouvé du thorium dans toutes les préparations d'actinium, parce qu'aussi l'actinium de Debierne semble se rapprocher souvent, mais non toujours, du thorium au point de rue chimique. Hais ce n'est pas là une raison. Ce n'est pas le corps temporairement actif el entrainé par le thorium qui est l'actinium, c'est le corps d'activité perrianente $^{*}$ possédant les propriétés radioaclives qu'on a signalées. Un produit qui perd son activité en quelques semaines ou quelques mois ne peut itre de l'actinium.

Jo crois que de semblables malentendus seront inévitables tant qu'on considérera les transformations radioactives au point de vue purement chimique. Il ne faut pas oublier que nous ne connaissons pas du tout les propriétés chimiques des corps radioactifs. Nous

\footnotetext{
* Cf. aussi réponse de Debierne à Marckwald dans 29.
}

arrivons ì les concentrer, mais par des procédés empiriques, et non par l'application de méthodes théoriques; les propriétés analytiques qui les caractérisent. leur solubilité par exemple, nous demeurent inconnues. On cite souvent comme propriété du Th S et de l'AcX le fait que ces corps sont solubles dans lammoniaque parce qu'ils restent dans la liqueur yuand on précipite le thorium ou l'actinium par l'ammoniaque. Mais le mot "soluble " ne doit pas ici être pris à la lettre. L'expérience n’apprend qu’une chose, saroir que le ThX et l'Ac $X$ ne sont pas intraines arec lhidrate précipité. Nais qu'il s'agisse lì d’une solubilité proprement dite, nous n'en savons rien. Nous sommes ici au-dessous de la limite des concentrations mesurables, el comparé aux solutions dont il s'agit, le sulfate de baryte par exemple est très soluble dans l'ean. Nème si le Th $X$ et l'Ac X éta ent rigoureusement insolubles dans l'ammoniaque, ils ne se précipiteraient pas arec les oxydes à moins d'ètre entrainés par euix, car nous ne possédons pas de filtre pour retenir les molécules isolées.

En ce qui concerne par exemple la séparation de l'Ac X par l'ammoniaque, Marckwald ${ }^{25}$ arait ajouté de l'ammoniaque à la solution de thorium renfermant son produit et ne trourait pas d'Ac $\mathrm{X}$ dans la liqueur filtrće. Mais cela ne prouve nullement que le thorium de Marckwald ne contienne pas d'Ac X. Cette expérience montre seulement que le produit actif entrainé par le thorium dans la précipitation par l'hrposulfite suit encore le thorium dans la précipitation par l'ammoniaque. La séparation par l'ammoniaque n'est pas une propriété analytique caractéristique de l'Ju $X$, car elle ne repose pas sur la ( non-précipitation ») mais sur le ( non-entrainement ) et l'entrainement varie selon la présence des impuretés.

La seule caracléristique de l'Ac X est l'ensemble de ses propriélés radioactives.

De nombreuses expériences sur l'activité artiliciollement induite sur les éléments, par exemple par précipitation, qu'on a expliquées aussi par l'entrânement des produits actifs, ont montré que le sens de ces réactions (sit vania verbo) dépend essentiellement non de la nature du solvant, mais de celle du corps prici_ pité en quantité pondérable. En présence de certains corps, certains produits sont entrainés dans la préc:pitation, mais non à proprement parler précipitís. Nous ne connaissons rien de leur's proprictés ahimiques.

Nais ce qu'on vient de dire des produits radioactifs est vrai aussi des substance's mires, tant quon les aura en trop petite quantité pour les soumettre à l'analyse chimique ou spectrale, comme cest le cas pour l'actinium et l'émanium. Yous ne pouvons parler des propriétés chimiques de ces corps ni les comparer, car nous n'en connaissons pas mìme une. La comparaison ne peut donc se faire quau point de rue 
radioactif, et elle conduit à l'identité des deux corps. Nous pourons donc dire que, quelques différences qu'on puisse observer entre les préparations de Giesel et celles de Debierne au point de vue chimique, spectroscopique, etc., il n'y en a pas au point de vue radioactif et cela suffit à affirmer l'identité de l'émanium et de l'actinium.

Traduil de l'allemand par Léon BLoon.

\section{Bibliographie.}

25. F. Gresen. Ueber die «Thor--1ktivitil 》 de's Monazits. Cikem. Ber., 38, 25\%ห-2506, 1905.

26. 1. In nirnes. Sur les gaz produits par l'actinium, C. R., 141, 585-585, 1905.

27. T. Cioctensis. On the alsorption of the $\beta$ and y-rays of Actinium, Phil. Ilag., 375-379, septembre 190\%.

28. 0. ILaнs. Ueber die neues in Emanation der Thorium gebendes radioaktives Element, Jahrb. d. Radioaht., 2, 255266,1905 .

29. A. Deвierxe. Ueber einige Eigenschaften des 1 ktiniums, Phys. Zeitschr., 7, 14-16, 1906.

go gio gio go gio 